\title{
Comparative studies of compatible and incompatible pepper-Tobamovirus interactions and the evaluation of effects of 24-epibrassinolide
}

\author{
A. JANECZKO ${ }^{*,+}$, M. DZIURKA*, G. GULLNER ${ }^{* *}$, M. KOCUREK ${ }^{* * *}$, M. RYS*, D. SAJA*, \\ A. SKOCZOWSKI", I. TÓBIÁS ${ }^{* *}$, A. KORNAS ${ }^{\#}$, and B. BARNA** \\ Polish Academy of Sciences, The Franciszek Górski Institute of Plant Physiology, Niezapominajek 21, \\ 30-239 Kraków, Poland* \\ Plant Protection Institute, Centre for Agricultural Research, Hungarian Academy of Sciences, Herman Ottó út 15, \\ 1022 Budapest, Hungary** \\ Institute of Biology, The Jan Kochanowski University, Świętokrzyska 15, 25-406 Kielce, Poland ${ }^{* * *}$ \\ Institute of Biology, Pedagogical University of Cracow, Podchorażych 2, 30-084 Kraków, Poland ${ }^{\#}$
}

\begin{abstract}
The aim of study was to gain a deeper knowledge about local and systemic changes in photosynthetic processes and sugar production of pepper infected by Obuda pepper virus (ObPV) and Pepper mild mottle virus (PMMoV). PSII efficiency, reflectance, and gas exchange were measured 48 and/or $72 \mathrm{~h}$ after inoculation (hpi). Sugar accumulation was checked $72 \mathrm{hpi}$ and $20 \mathrm{~d}$ after inoculation (as a systemic response). Inoculation of leaves with ObPV led to appearance of hypersensitive necrotic lesions (incompatible interaction), while PMMoV caused no visible symptoms (compatible interaction). ObPV (but not PMMoV) lowered $F_{v} / F_{m}$ (from 0.827 to 0.148 at $72 \mathrm{hpi}$ ). Net photosynthesis decreased in ObPV-infected leaves. In ObPV-inoculated leaves, the accumulation of glucose, fructose, and glucose-6-phosphate was accompanied with lowered sucrose, maltoheptose, nystose, and trehalose contents. PMMoV inoculation increased the contents of glucose, maltose, and raffinose in the inoculated leaves, while glucose-6-phosphate accummulated in upper leaves.
\end{abstract}

Additional key words: brassinosteroids; $\mathrm{CO}_{2}$ assimilation; systemic virus response; water band index; xanthophyll cycle.

\section{Introduction}

Peppers (Capsicum spp.) are very important vegetables worldwide. However, the number of virus species infecting pepper crops and their incidences has increased considerably over the past 30 years. Viral diseases can seriously reduce the yield and quality of all pepper cultivars. Tobamovirus, as an important genus of pepper viruses, are mechanically distributed and occur practically all over the word. Several viruses belonging to the genus Tobamovirus are major pathogens for pepper plants. Resistance conferred by the $L^{3}$ gene is very efficient against most Tobamoviruses except for some closely related PMMoV isolates, which are able to overcome this type of resistance and cause systemic infection with mild mottle symptoms on leaves and spreading of the virus into the whole plant (Tóbiás et al. 1989, Velasco et al. 2002). On the other hand, ObPV, which also belongs to the genus Tobamovirus, can not break the $L^{3}$ gene-mediated resistance, and this virus elicits a wide array of defense mechanisms including the hypersensitive response (HR) accompanied by cell death and the development of necrotic lesions.

Viral infections cause various changes in crucial plants

Received 1 August 2016, accepted 20 February 2017, published as online-first 8 April 2017.

${ }^{+}$Corresponding author; e-mail: ania@belanna.strefa.pl

Abbreviations: BR - brassinosteroid; Chl - chlorophyll; dpi - days after inoculation; ETC - electron transport chain; hpi - hours after inoculation; ObPV - Obuda pepper virus; PMMoV - Pepper mild mottle virus; PRI - physiological reflectance index; RC - reaction center; ROS - reactive oxygen species; RWC - relative water content; SIPI - structure insensitive pigment index; UDP - uracildiphosphate glucose; WBI - water band index; 24-epi-BR - 24-epibrassinolide;.

Acknowledgment: We thank Dr. Lajos Zatykó (Research Institute of Vegetable Crops, Budatétény, Hungary) for kindly providing the pepper seeds. The experiments were conducted within a bilateral cooperation project between the Polish and Hungarian Academy of Sciences during 2013-2016. The financial support of the Hungarian Scientific Research Fund (OTKA K83615) is gratefully acknowledged.

(C) The Author(s). This article is published with open access at link.springer.com 
processes, such as photosynthesis, sugar metabolism, and respiration (Balachandran et al. 1994, Ryslava et al. 2003). As for light reactions of photosynthesis, virus infections affect the efficiency of photosystems, which is mainly connected to the ultrastructure and functionality of chloroplasts. Viral pathogens can affect the number, size, and morphology of chloroplasts, in addition, they influence the size and number of chloroplast inclusions, e.g. of starch grains (Almási et al. 2001). The oxygen-evolving complex (OEC) of the photosynthetic electron transport chain was identified as the main target of either the Italian or the Spanish strain of PMMoV (PMMoV-I and PMMoV-S, respectively) in Nicotiana benthamiana (Rahoutei et al. 1999, 2000, Pérez-Bueno et al. 2004). Analysis of chlorophyll (Chl) fluorescence induction curves (slow and fast Chl $a$ fluorescence kinetic) is a very popular method to estimate the effectiveness of photosystems (Papageorgiou et al. 2007). This method has been already used during studies devoted to virus-plant interactions (Baron et al. 1995, Gonçalves et al. 2005, Rys et al. 2014). Methods allowing the measurement of leaf reflectance are less popular but also useful for the study of virus-plant interactions, for example for an early detection of viral infection in tomato (Krezhova et al. 2014). The low reflectance of solar radiation by leaves in the photosynthetically active region (PAR, 400-700 nm) is mainly linked to absorption by Chls and other pigments connected to harvesting of light energy (Knipling 1970, Solovchenko 2010). Once the relation between reflectance and pigment concentration has been established by typical biochemical techniques, reflectance analysis can provide a quite inexpensive, fast and nondestructive assay of various photosynthetic pigments. Since the amount of foliar pigments, mainly Chls and carotenoids (Car), are affected by a variety of stress factors (Peñuelas et al. 1994), reflectance analysis can also provide a mean of assessing the degree to which plants are affected by abiotic and biotic stresses. Furthermore, the leaf reflectance at $970 \mathrm{~nm}$ was used to define the leaf water balance index, which provides information about changes in the relative water content (RWC), the leaf water potential, and the stomatal conductance (Peñuelas et al. 1993, Peñuelas et al. 1997). In another words, the reflectance measurement can be a complementary method to fluorescence measurements; it allows obtaining additional information about antenna pigments building PS (including pigment changes in the xanthophyll cycle) and leaf water management.

Viral infection may also affect the light-independent (dark) phase of photosynthesis connected to $\mathrm{CO}_{2}$ assimilation as well as the sugar production in host plants. Sugars as main products of photosynthesis are however not only nutrients needed for growth, respiration, and the accumulation of storage compounds, but they can serve as signals in the regulation of gene expression (Koch 1996). Sugar signals can significantly contribute to immune responses against pathogens by activating defense genes (Herbers et al. 1996) and they can function as priming molecules in infected plants (Moghaddam and Van den Ende 2012). Among sugars, sucrose is emerging as a candidate for signalling molecule in plant innate immunity (GómezAriza et al. 2007).

The extracellular cell wall invertase (CWI) enzymes, which split sucrose into glucose and fructose, are also considered as pathogenesis-related (PR) proteins (Roitsch et al. 2003). The efficiency of light and carbon reactions of photosynthesis are controlled by plant hormones, such as abscisic acid (ABA), cytokinins or the plant steroid hormones - brassinosteroids (BRs), which can modulate the biochemical processes allowing flexible acclimation to changing environmental conditions. In recent years, our knowledge significantly increased about BRs and their regulatory roles during photosynthesis. BRs increase the content of photosynthetic pigments and induce the activity of several enzymes, such as carbonic anhydrase, phosphoenolpyruvate carboxylase, Rubisco, as well as enzymes involved in sugar production and transport (Holá 2011). BRs stimulate also the expression of genes encoding Calvin cycle enzymes and Rubisco activase (Holá 2011). The importance of BRs in the regulation of photosynthesis has been confirmed by using BR-deficient mutants or mutants with a disturbed BR signalling (Holá 2011, Janeczko et al. 2016). Stress factors can enhance the effect of BRs on photosynthesis. However, most research projects have been focused on abiotic stress, and very limited information is available about the mechanism of action of BRs on photosynthesis in infected plants (Skoczowski et al. 2011). Nevertheless, BR possess multidirectional activity not only directly connected to process of photosynthesis. As a result of incompatible pathogen infection, plants shift metabolism from normal growth to defense responses called microbe- or pathogeninduced immunity (Wang 2012), which is often accompanied by hypersensitive cell death. The hypersensitive response of plants can be alleviated by BR treatment (Skoczowski et al. 2011). In addition, the leucine-rich repeat receptor kinase (LRR-RK) BAK1 is probably involved in the process; it serves as a co-receptor for ligand binding LRR-RKs for steroid hormones and microbeassociated molecular patterns (MAMPs), including BRI1 (binding brassinosteroids) and FLS2, the receptor of bacterial flagellin peptide activating microbe-induced immunity (Wang 2012). Moreover, BR application increases cell capacity to deal with reactive oxygen species (ROS), of which the excessive amount is generated during pathogen infection, stabilizes cell membranes, and regulates biosynthesis of other hormones, among others important stress hormone - ABA (Yusuf et al. 2014, Janeczko et al. 2016).

Since data in the literature on photosynthesis and sugar content in virus-infected resistant plants with hypersensitive lesions are very limited, the aim of our present study was to gain a deeper knowledge about local and systemic changes in the efficiency of the light reaction of photosynthesis as well as in the $\mathrm{CO}_{2}$ assimilation and sugar 
production in pepper infected by ObPV (incompatible interaction) and PMMoV (compatible interaction). Furthermore, we wanted to answer the question whether viruselicited negative changes in plant photosynthetic processes (PSII efficiency and sugar accumulation) can be alleviated by plant steroid hormones (BRs). Our investigations provide additional information which may help to explain

\section{Materials and methods}

Plant culture, experimental design and sample collection: Seeds of the pepper (Capsicum annuum L., cultivar TL 1791) harboring the $L^{3}$ resistance gene were planted into soil and grown under greenhouse conditions $\left[23^{\circ} \mathrm{C}\right.$; photoperiod of $16 \mathrm{~h}$, irradiance of $160 \mu \mathrm{mol}$ (photon) $\mathrm{m}^{-2} \mathrm{~s}^{-1}$; relative humidity of $75-80 \%$ ]. Two-month-old plants were divided into five groups (10 plants per one group) in order to carry out different treatments:

(1) Plants were inoculated with ObPV (the ObPV strain was isolated in Hungary, formerly used synonym: $\mathrm{Ob}$ strain of Tomato mosaic virus).

(2) Plants were inoculated with $L^{3}$-resistance breaking strain of PMMoV (isolated in USA, formerly used synonym: Samsun latent strain of Tobacco mosaic virus) (Tóbiás et al. 1989, Rys et al. 2014).

Both viral inoculations were made with cellit as an abrasive.

(3) MOCK control - leaves were inoculated with cellit and buffer but without any virus to test the effect of the slight mechanical injury caused during the virus inoculation.

(4) Plants were pretreated (sprayed) with $100 \mathrm{nM}$ 24-epibrassinolide (24-epi-BR) two days before ObPV inoculation.

(5) Plants were sprayed with $100 \mathrm{nM}$ 24-epi-BR two days before PMMoV inoculation.

Virus inoculations were carried out on the fifth and sixth true leaves as described earlier (Rys et al. 2014).

Noninvasive measurements were carried out on ObPV, PMMoV, and MOCK-inoculated control leaves of pepper plants. Fast kinetic measurements of Chl $a$ fluorescence (JIP test) were carried out 48 and $72 \mathrm{~h}$ after inoculation (hpi) on fifth and sixth leaves. Fluorescence measurements were carried out also on the systemic, uninfected upper leaves of virus-inoculated plants at $15 \mathrm{dpi}$. Gas-exchange and reflectance coefficients were measured on the fifth and sixth leaves at 48 hpi. For sugar analyses, samples were taken from the fifth and sixth leaves $72 \mathrm{hpi}$ and additionally from uninfected upper leaves $20 \mathrm{dpi}$ in order to characterize the systemic defense response. In the case of 24-epi-BR-pretreated and virus-inoculated plants, only $\mathrm{Chl} a$ fluorescence measurements and sugar analyses were carried out.

Fast kinetics of Chl $\boldsymbol{a}$ fluorescence (JIP test): The fast fluorescence kinetics of Chl $a$ was measured in darkadapted leaves by using a PEA fluorometer (Hansatech Ltd., King's Lynn, Norfolk, England) as described by the mechanisms of hypersensitive defense reaction of pepper plants against viral pathogens and the role of steroid hormones in defense processes. In addition, by using noninvasive methods to characterize the efficiency of photosynthesis, we may detect metabolic disorders before the appearance of visible symptoms of virus infection, which could be of practical importance.

Skoczowski et al. (2011). From the fluorescence curves for each sample (leaf), the following technical parameters were extracted and/or calculated: $\mathrm{F}_{\mathrm{o}}$ - initial fluorescence at $50 \mu \mathrm{s} ; \mathrm{F}_{\mathrm{m}}-$ maximal fluorescence; $\mathrm{F}_{\mathrm{v}}$ - variable fluorescence $\left(F_{m}-F_{o}\right)$; ratios of $F_{v} / F_{m}$ and $F_{v} / F_{o} ; S_{m}$ - normalized area $\left[\mathrm{S}_{\mathrm{m}}=\left(\right.\right.$ area between fluorescence curve and $\left.\mathrm{F}_{\mathrm{m}}\right) /$ $\left.\left(\mathrm{F}_{\mathrm{m}}-\mathrm{F}_{\mathrm{o}}\right)\right] ; \mathrm{M}_{\mathrm{o}}-$ normalized value of the initial slope $\left[\mathrm{M}_{\mathrm{o}}=4\left(\mathrm{~F}_{300 \mu \mathrm{s}}-\mathrm{F}_{\mathrm{o}}\right) /\left(\mathrm{F}_{\mathrm{m}}-\mathrm{F}_{\mathrm{o}}\right)\right.$, where $\mathrm{F}_{300 \mu \mathrm{s}}$ - fluorescence at $300 \mu \mathrm{s}$ ]; $\mathrm{N}$ - turnover number of $\mathrm{Q}_{\mathrm{A}}$ (primary quinone acceptor) from time 0 to time, when $F_{m}$ is reached $\left[\mathrm{N}=\mathrm{S}_{\mathrm{m}} \times \mathrm{M}_{\mathrm{o}} \times\left(1 / \mathrm{V}_{\mathrm{J}}\right)\right.$, where $\left.\left.\mathrm{V}_{\mathrm{J}}=\left(\mathrm{F}_{2 \mathrm{~ms}}-\mathrm{F}_{\mathrm{o}}\right) / \mathrm{F}_{\mathrm{m}}-\mathrm{F}_{\mathrm{o}}\right)\right]$; $\mathrm{V}_{\mathrm{K}}$ - relative fluorescence at $\mathrm{F}_{300 \mu \mathrm{s}},\left[\mathrm{V}_{\mathrm{K}}=\left(\mathrm{F}_{300 \mu \mathrm{s}}-\mathrm{F}_{\mathrm{o}}\right) /\right.$ $\left.\left(\mathrm{F}_{\mathrm{m}}-\mathrm{F}_{\mathrm{o}}\right)\right]$. Based on technical parameters, the quantum efficiencies were calculated: $\varphi_{\text {Po }}-$ maximum yield of primary photochemistry (relation of the energy transmitted to $\mathrm{RC}$ and the absorbed energy); $\psi_{\mathrm{o}}$ - probability that a trapped exciton moves an electron into the ETC further than $\mathrm{Q}_{\mathrm{A}}{ }^{-}$(the relation of energy transferred to ETC and the energy transmitted to $\mathrm{RC}$ ); $\varphi_{\mathrm{E} o}$ - maximum yield of electron transport (the relation between the energy transferred to ETC and the absorbed energy). The equations for these parameters are from Strasser et al. (2000). Measurements were done in ten repetitions per treatment (one repetition $=$ one leaf from one plant).

Leaf spectral reflectance and calculation of reflection indices: Reflection measurements were carried out by using a CI-710 miniature leaf spectrometer (CID BioScience, Camas, WA, USA) on the top side of leaf lamina. Signal integration time was set to $300 \mathrm{~ms}$ with smoothing factor (boxcar) equal to 10. Each measurement was the average from ten scans. The results were plotted as reflection intensity curves as a function of wavelength. Reflection ratios were calculated automatically based on the following formulas: WBI $=\mathrm{R}_{900} / \mathrm{R}_{970}$ (Peñuelas et al. 1997); $\mathrm{SIPI}=\left(\mathrm{R}_{800}-\mathrm{R}_{435}\right) /\left(\mathrm{R}_{415}+\mathrm{R}_{435}\right)$ (Peñuelas et al. $1995 \mathrm{a}) ; \mathrm{PRI}=\left(\mathrm{R}_{531}-\mathrm{R}_{570}\right) /\left(\mathrm{R}_{531}+\mathrm{R}_{570}\right)$ (Peñuelas et al. 1995b). Structure-insensitive pigment index (SIPI) is associated with the ratio of total carotenoids to $\mathrm{Chl} a$ (Peñuelas et al. 1995a). The physiological reflectance index (PRI) is connected with changes in the reflectance intensity around $531 \mathrm{~nm}$ and informs about pigment changes in the xanthophyll cycle (Gamon et al. 1992), which often accompany stress (Gamon et al. 1997). The reflectance at $970 \mathrm{~nm}$ was used to define the water band index (WBI). WBI has been used to track changes in relative water content (RWC), leaf water potential, and 
stomatal conductance (Peñuelas et al. 1993). As for stress detection, WBI is a good indicator of the water status in plants exposed to salinity stress (Peñuelas et al. 1997). Measurements were done in ten repetitions per treatment (one repetition $=$ one leaf from one plant).

Gas exchange: Leaf gas exchange was measured by an infrared gas analyzer Li-6400 (Licor Inc., Lincoln, NE, USA) equipped with a standard leaf chamber and a LED light source (6400-02). Conditions of measurements: PAR $=1,000 \mu \mathrm{mol}$ (photon) $\mathrm{m}^{-2} \mathrm{~s}^{-1}$; constant temperature of the measurement block: $30^{\circ} \mathrm{C}$; controlled $\mathrm{CO}_{2}$ supply: $400 \mu \mathrm{mol}\left(\mathrm{CO}_{2}\right) \mathrm{mol}^{-1}$ (air); relative humidity: $35-40 \%$. The transpiration rate $(E)$, intercellular $\mathrm{CO}_{2}$ concentration $\left(C_{\mathrm{i}}\right)$, stomatal conductance $\left(g_{\mathrm{s}}\right)$, and net photosynthetic rate $\left(P_{\mathrm{N}}\right)$ were measured. Measurements were performed in seven replicates, where one replicate was one leaf from each plant.

Soluble sugars: Qualitative and quantitative analysis of soluble sugars was performed according to procedure

\section{Results}

Symptoms of virus inoculations: Following ObPV inoculation the first visible symptoms (hypersensitive necrotic lesions) appeared $72 \mathrm{hpi}$, in accordance with earlier studies (Rys et al. 2014). Pretreatment with $100 \mathrm{nM}$ 24-epi-BR significantly alleviated the visible symptoms of ObPV inoculation (Fig. 1). None or very mild symptoms appeared on PMMoV-inoculated leaves. Earlier studies showed that the replication rate of PMMoV was dramatically higher in the inoculated pepper leaves than that of ObPV (Juhász et al. 2015). On the other hand, PMMoV inoculation resulted in slight systemic chlorotic symptoms on the upper leaves between 14 and 20 dpi. However, the 24-epi-BR treatment did not change the visible symptoms on control, noninfected, or PMMoV-infected plants.

PSII efficiency in virus-inoculated pepper leaves: Measurements of fast kinetics fluorescence revealed a described by Janeczko et al. (2010). Fresh leaf samples $(0.3 \mathrm{~g})$ were lyophilized, ground, and weighed into aliquots ( $5 \mathrm{mg}$ of dry mass) for extraction with deionised water. After dilution with acetonitril $(1 / 1, \mathrm{v} / \mathrm{v})$ sugars were separated by means of high performance anion-exchange chromatography with pulse amperometric detection (Agillent 1200 with ESA coulochem2, USA). Identification and quantitation were based on results acquired for pure standards of studied sugars (Sigma-Aldrich, Poznań, Poland) under the same conditions as that for samples. The analyses were performed in three replicates.

Statistical analysis of the results was carried out using the Statistica 10 (StatSoft, USA) program. Number of samples/repetitions is given in chapters dedicated to particular measurements. Statistical significance in Table 1 and in Figs. 2-5 was based on a Duncan's test $(P \leq 0.05)$. Statistical significance in Fig. 6 was estimated by the Student's test $(P=5 \%$ or $1 \%)$. Data presented on table and figures are mean values with standard deviations ( \pm SD).

significant impact of virus infections on the performance of pepper photosynthetic apparatus (Table 1, Fig. 2). The $F_{o}$ values determined 48 hpi and 72 hpi did not differ significantly between MOCK and virus-inoculated leaves (Table 1). ObPV inoculation and ObPV inoculation combined with 24-epi-BR pretreatment markedly decreased the $F_{v}$ values measured at 48 hpi (by 47 and $36 \%$, respectively) (Table 1). The decrease of $F_{v}$ values by ObPV and 24-epi-BR + ObPV treatments was even stronger at 72 hpi (by 92 and 57\%, respectively). PMMoV inoculation brought about only a slight, insignificant decrease of $\mathrm{F}_{\mathrm{v}}$ values, and the pretreatment with 24-epi-BR induced further a slight decrease of this parameter (Table 1). ObPV inoculation and ObPV inoculation combined with 24-epi-BR pretreatment significantly decreased also the $\mathrm{F}_{\mathrm{m}}$ values measured 48 hpi (by 38 and 29\%, respectively) (Table 1). The decrease of $F_{m}$ values was even stronger at 72 hpi (by 77 and 48\%, respectively). PMMoV
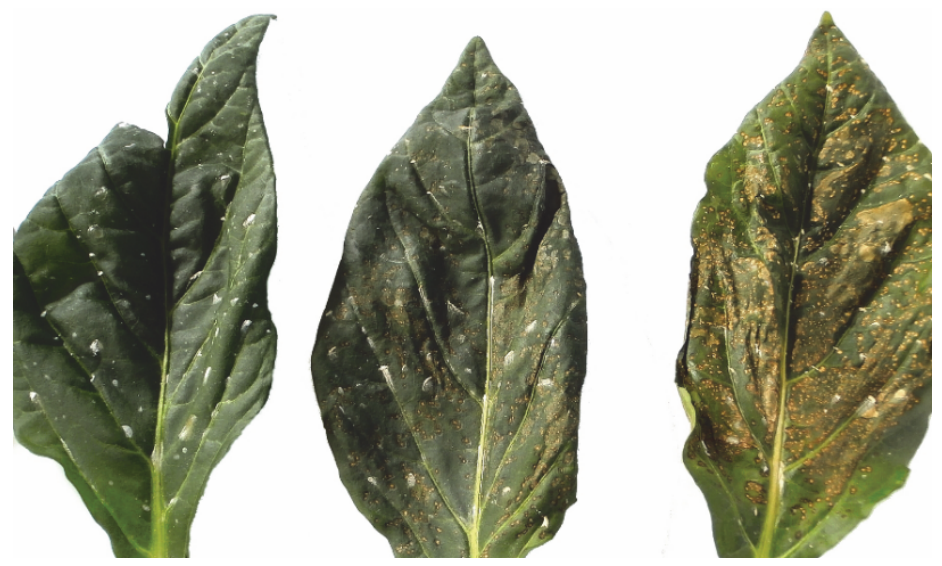

Fig. 1. Visual symptoms on pepper leaves following MOCK (control) inoculation (on the left), 3 days after ObPV inoculation (on the right) or after pretreatment with $100 \mathrm{nM}$ 24-epibrassinolide (24-epi-BR) and 2 days later a subsequent ObPV inoculation (in the middle). Photos were taken of the $6^{\text {th }}$ leaves. The white spots on leaves are cellit spots. 


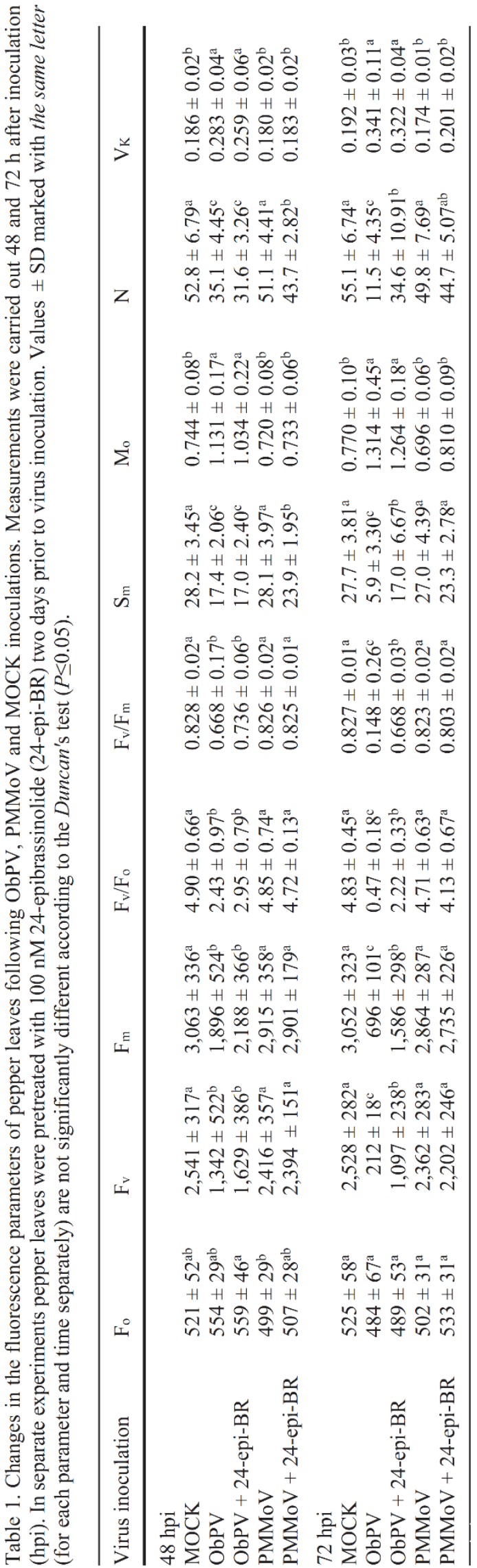

inoculation did not modify the $\mathrm{F}_{\mathrm{m}}$ values (Table 1). As a consequence of decreasing $F_{v}$ values, the $F_{v} / F_{o}$ values were also markedly lower in ObPV-inoculated leaves $48 \mathrm{hpi}$ (by $51 \%$ ), whereas in 24-epi-BR pretreated and ObPVinoculated leaves, the rate of decrease was $40 \%$ (Table 1). Infection with PMMoV had no effect on $F_{v} / F_{o}$ values. ObPV (but not PMMoV) lowered $F_{v} / F_{m}$ significantly after 48 and 72 hpi. ObPV inoculation led to a strong decrease also in the $\mathrm{S}_{\mathrm{m}}$ values 48 hpi (by 38\%) and 72 hpi (by 79\%) (Table 1). Pretreatment with 24-epi-BR alleviated this effect significantly at $72 \mathrm{hpi}$. ObPV inoculation markedly increased the $\mathrm{M}_{\mathrm{o}}$ values both at 48 hpi and 72 hpi (Table 1) and the 24-epi-BR pretreatment did not counteract this effect. Infection with PMMoV had no effect on $\mathrm{M}_{\mathrm{o}}$ values. On the other hand, ObPV inoculation resulted in lower $\mathrm{N}$ values 48 hpi (by 34\%) and 72 hpi (79\%) (Table 1). Pretreatment with 24-epi-BR alleviated this effect significantly 72 hpi. Infection with PMMoV alone had a mild effect on $\mathrm{N}$ values although interestingly 24-epi-BR pretreatment significantly lowered the $\mathrm{N}$ values in PMMoVinfected leaves. ObPV inoculation markedly elevated the $\mathrm{V}_{\mathrm{K}}$ values in pepper leaves both $48 \mathrm{hpi}$ and $72 \mathrm{hpi}$, while PMMoV had no effect on this parameter. Furthermore, in ObPV-inoculated leaves, the $\varphi_{\mathrm{Po}}, \psi_{\mathrm{o}}$, and $\varphi_{\mathrm{E}}$ values determined 48 hpi were substantially lower than those measured in MOCK-inoculated control leaves (by 19, 27, and $40 \%$, respectively) (Fig. $2 A$ ). Pretreatment with $24-$ epi-BR markedly alleviated the negative effect of ObPV infection 72 hpi (Fig. 2B). Generally, the compatible PMMoV infection had mild effect on PSII efficiency and BR pretreatment did not change the $\varphi_{\text {Po }}, \psi_{\mathrm{o}}$, and $\varphi_{\text {Eo }}$ values.

Measurements of fast kinetics of Chl $a$ fluorescence in the systemic, noninoculated upper leaves of virus-infected plants did not demonstrate any significant impact of either ObPV nor PMMoV inoculations on the performance of the photosynthetic apparatus at $15 \mathrm{dpi}$ (data not shown). No significant changes were noticed neither in technical parameters of fluorescence nor in quantum efficiencies characterizing PSII efficiency. In addition, pretreatment with $100 \mathrm{nM}$ 24-epi-BR exerted no significant effect on the PSII efficiency of systemic, noninoculated upper leaves (data not shown).

Leaf reflectance: Both ObPV and PMMoV inoculations strongly increased the leaf reflection of radiation in pepper leaves as compared to MOCK-inoculated leaves at $48 \mathrm{hpi}$ (Fig. 3A). This effect was particularly noticeable in the PAR region. Based on reflectance spectra, the SIPI, PRI, and WBI indices were calculated. ObPV inoculation significantly decreased the SIPI, PRI, and WBI values at $48 \mathrm{hpi}$ by approx. 20,70 , and $6 \%$, respectively (Fig. $3 B-D$ ). The analysis of WBI values showed that ObPV inoculation slightly, but statistically significantly, diminished the hydration of pepper leaves (Fig. 3D). In contrast to ObPV, $\mathrm{PMMoV}$ inoculation altered significantly only the SIPI value, which was approx. $20 \%$ lower than that in the MOCK-inoculated, control leaves at 48 hpi (Fig. 3B). 


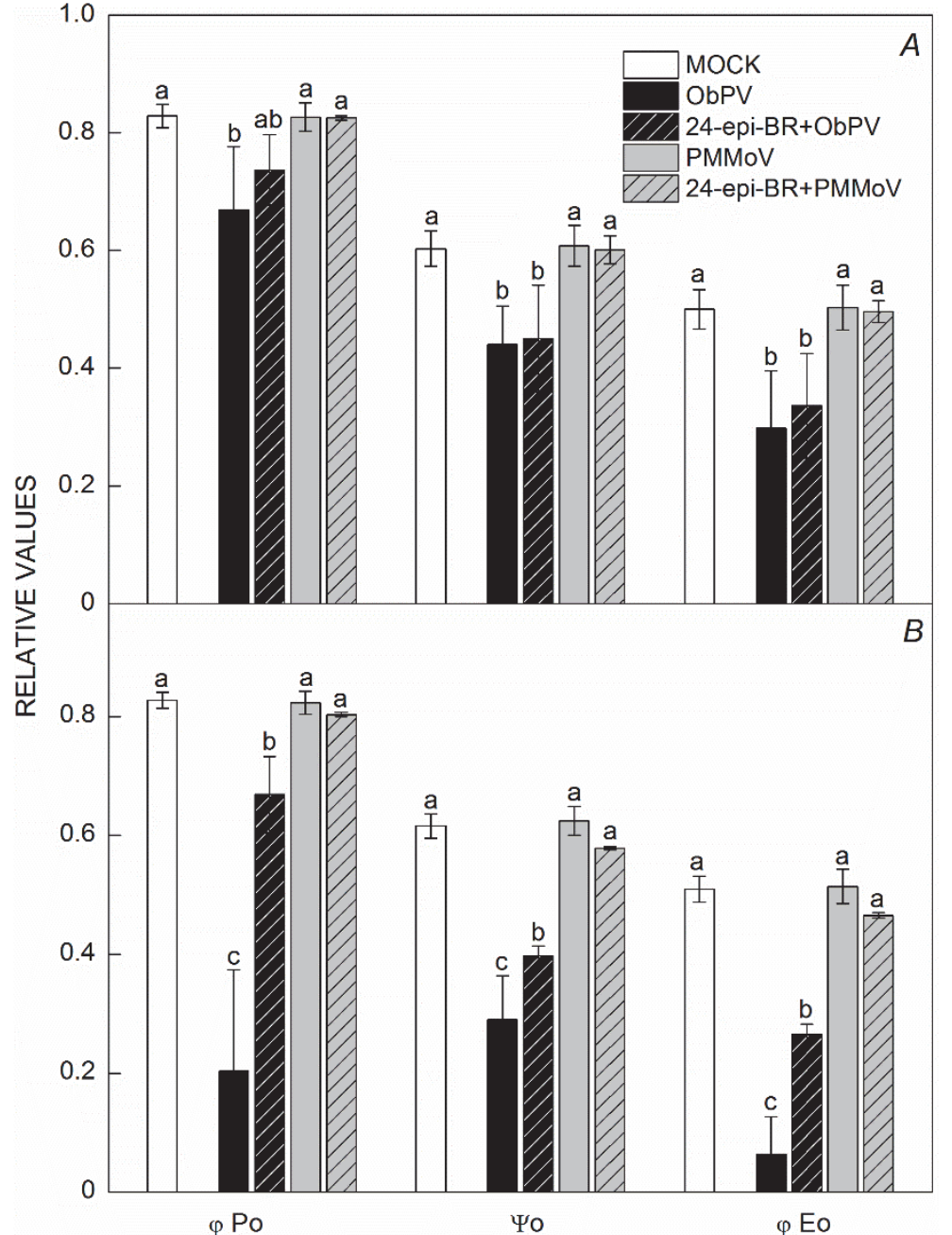

Fig. 2. Quantum efficiencies characterizing PSII in pepper leaves following ObPV, PMMoV, and MOCK (control) inoculation $48(A)$ and 72 hours $(B)$ after inoculation (hpi), with or without a pretreatment with $100 \mathrm{nM}$ 24-epibrassinolide (24-epi-BR). Values marked with the same letter (for each parameter and time period separately) are not significantly different according to Duncan's test $(P \leq 0.05)$. $\varphi_{\mathrm{Po}}-$ maximum yield of primary photochemistry; $\psi_{\mathrm{o}}$ - probability that a trapped exciton moves an electron into the electron transport chain (ETC) further than $\mathrm{Q}_{\mathrm{A}^{-}}$; $\varphi E_{0}-$ maximum yield of electron transport.
Leaf gas exchange: ObPV inoculation massively decreased the $P_{\mathrm{N}}$ (by $95 \%$ ), the $g_{\mathrm{s}}$ (by $70 \%$ ), and the $E$ (by $70 \%$ ), while markedly elevated the $C_{\mathrm{i}}$ (by $20 \%$ ) of pepper leaves at 48 hpi (Fig. 4). In contrast to ObPV, PMMoV inoculation did not change significantly any of these parameters (Fig. 4).

Foliar sugar contents: The foliar contents of ten different sugars (fructose, glucose, glucose-6-phosphate, 1-kestose, maltoheptose, maltose, nystose, raffinose, sucrose, and trehalose) and two sugar alcohols (inositol and glycerol) were measured in ObPV-, PMMoV- and MOCK-inoculated control pepper plants. We analyzed the changes in the contents of sugars and sugar alcohols both in the virusinoculated lower leaves (local effect, $72 \mathrm{hpi}$ ) and in the upper, noninoculated systemic leaves (systemic effect, 20 dpi) of the ObPV- or PMMoV-inoculated pepper plants. After ObPV inoculation a massive increase in glucose ( 5.5 fold) and fructose ( 8.4 fold) was observed in the inoculated lower leaves, while the sucrose content decreased by about $50 \%$ (Fig. $5 A-C$ ). In addition, ObPV inoculation markedly decreased the amount of trehalose, nystose, and maltoheptose in the infected leaves, while that of glucose-6-phosphate massively increased (the presence of glucose-6-phosphate was below detection limit in MOCK-inoculated leaves) (Fig. 5E,H,K,L). PMMoV infection slightly but significantly increased only the contents of glucose, maltose, and raffinose in the inoculated leaves (Fig. 5A,D,F). The foliar contents of 1-kestose, inositol, and glycerol did not change significantly neither by ObPV nor by PMMoV inoculation at any leaf position (Fig. $5 G, I, J$ ). Sugar contents in the uninfected upper, younger leaves were generally higher as compared to lower, inoculated leaves. The sugar contents did not change significantly in the upper, noninfected systemic leaves neither by ObPV nor by PMMoV inoculation $20 \mathrm{dpi}$, except only glucose-6-phosphate, the content of which was strongly elevated by both viruses (Fig. 5).

The effect of a pretreatment (spraying) with $100 \mathrm{nM}$ 24-epi-BR two days prior ObPV inoculation was also examined on the foliar sugar contents at $72 \mathrm{hpi}$ (local effect) and $20 \mathrm{dpi}$ (systemic effect). The massive increase of glucose contents in ObPV-infected leaves were only partly alleviated by 24-epi-BR at $72 \mathrm{hpi}$ (Fig. 6). Interestingly the foliar contents of glucose was lower, while of maltoheptose and nystose were significantly 

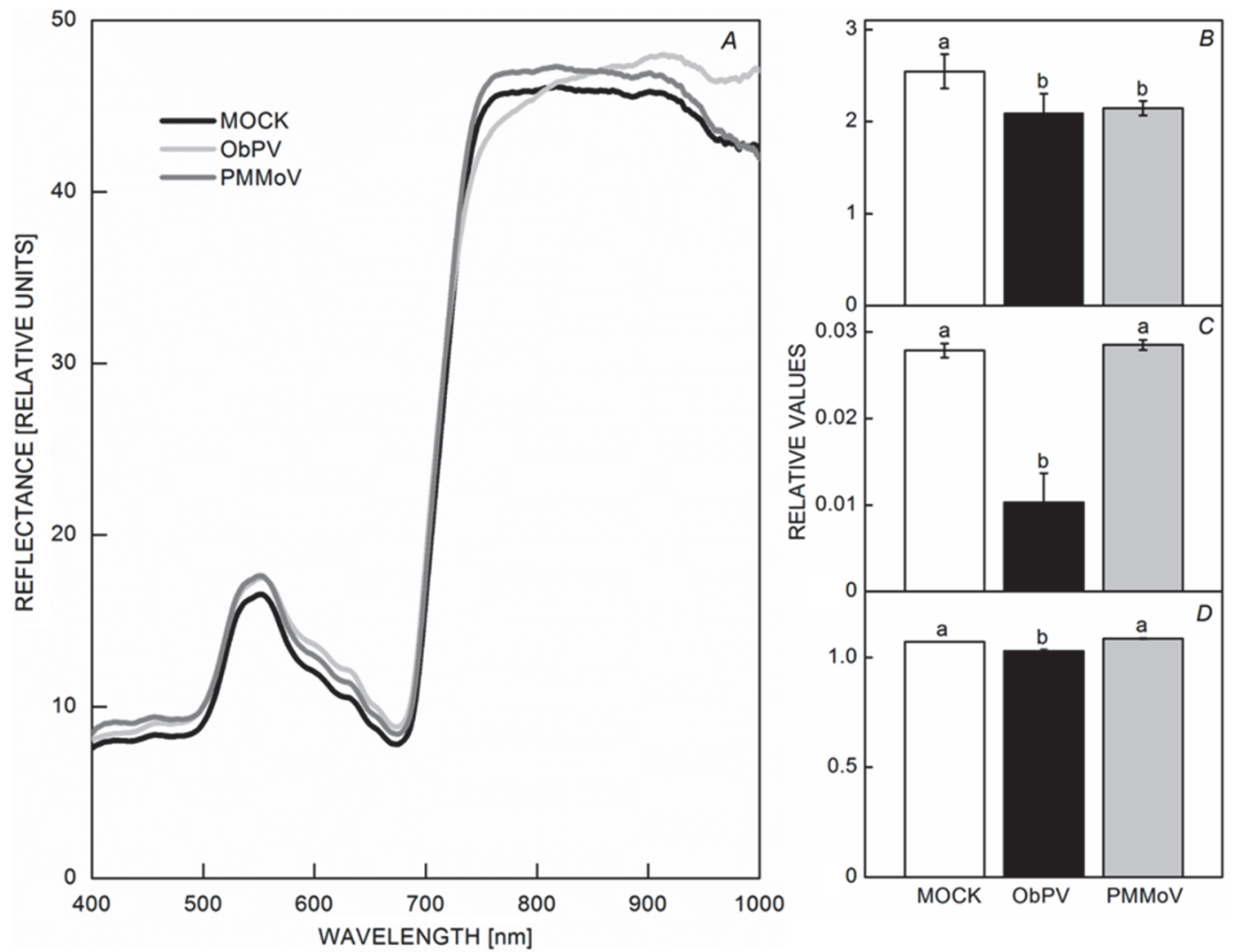

Fig. 3. Reflectance signature $(A)$ and selected spectral reflectance indices $(B, C, D)$ describing physiological properties of pepper leaves following ObPV, PMMoV, and MOCK (control) inoculation 48 hours after inoculation (hpi). (B) Structure-insensitive pigment index $(\mathrm{SIPI}) ;(C)$ physiological reflectance index (PRI); $(D)$ water band index (WBI). Values marked with the same letter are not significantly different according to Duncan's test $(P \leq 0.05)$.

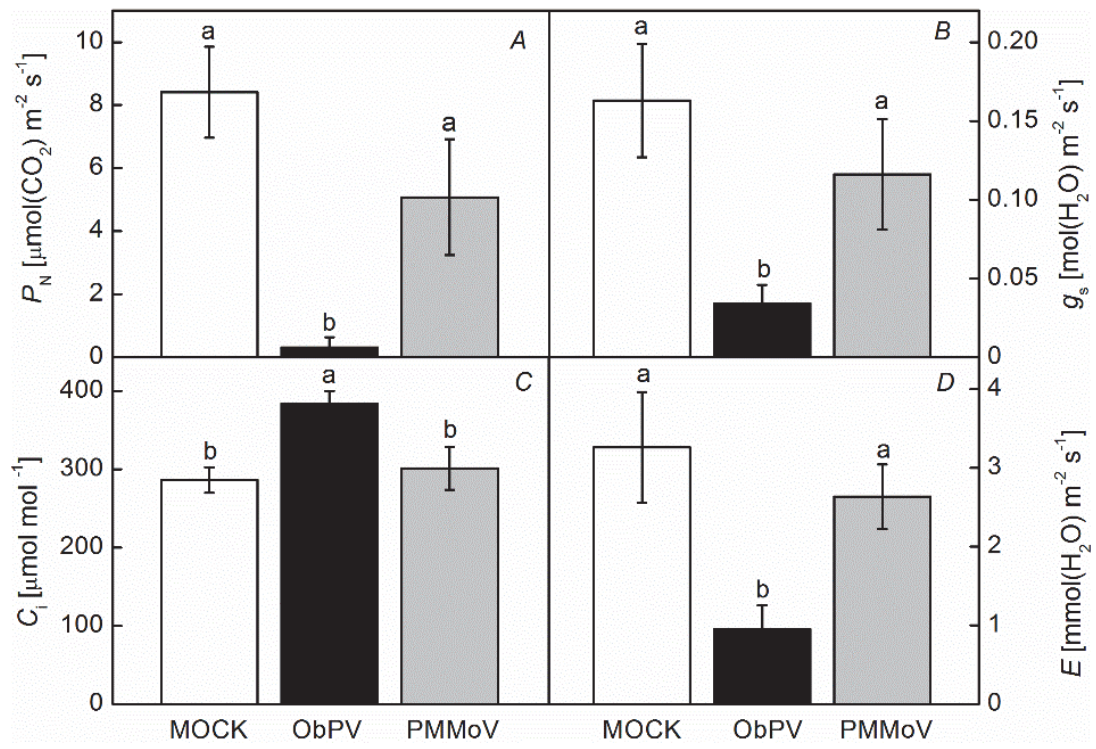

Fig. 4. Changes in the gas-exchange parameters of pepper leaves following ObPV, PMMoV, and MOCK (control) inoculation 48 hours after inoculation (hpi). $(A)-$ net photosynthetic rate $\left(P_{\mathrm{N}}\right) ;(B)$ - stomatal conductance $\left(g_{\mathrm{s}}\right) ;(C)$ - intercellular $\mathrm{CO}_{2}$ concentrations $\left(C_{\mathrm{i}}\right) ;(D)$ - transpiration rate $(E)$. Values marked with the same letter are not significantly different according to Duncan's test $(P \leq 0.05)$. higher in the 24-epi-BR-pretreated leaves 72 hpi than in those without this pretreatment, which shows that 24-epiBR counter-balanced (at least partly) the sugar contentchanging effect of ObPV inoculation (Fig. 6). None of the changes in other sugar contents were significant as a consequence of 24-epi-BR pretreatment (Fig. 6). In the upper, noninfected systemic leaves, the sugar contents were not modified significantly after a pretreatment with 100 nM 24-epi-BR (data not shown). 


\section{Discussion}

Physiological (photosynthetic) properties and growth of plants infected by virus have been shown to be negatively influenced by several researchers (Ryslava et al. 2003, Funayama et al. 1997, Guo et al. 2003). Furthermore, sugars directly or indirectly control a wide range of processes, including photosynthesis, sugar transport itself, nitrogen uptake, defense reactions, secondary metabolism, and hormonal balance, in addition to their role as a supply of energy and carbon backbone for growth and development in plants (Smeekens et al. 2010). However, information on the effect of virus infection on photosynthesis and carbohydrate metabolisms on a hypersensitive host plant is limited, therefore a hypersensitive incompatible and a systemic compatible interactions were compared in our work.

Generally, as for photosynthesis in our experiment, reaction of plants to ObPV virus was different from response to PMMoV. Response of plants to ObPV was manifested in tissue damage (necrosis), which is characteristic for hypersensitive reaction. Damages of leaf tissues are main cause of lowering photosynthetic efficiency (especially net photosynthesis but also PSII efficiency and other parameters). PMMoV remained latent and no symptoms/cell death appeared on inoculated leaves.

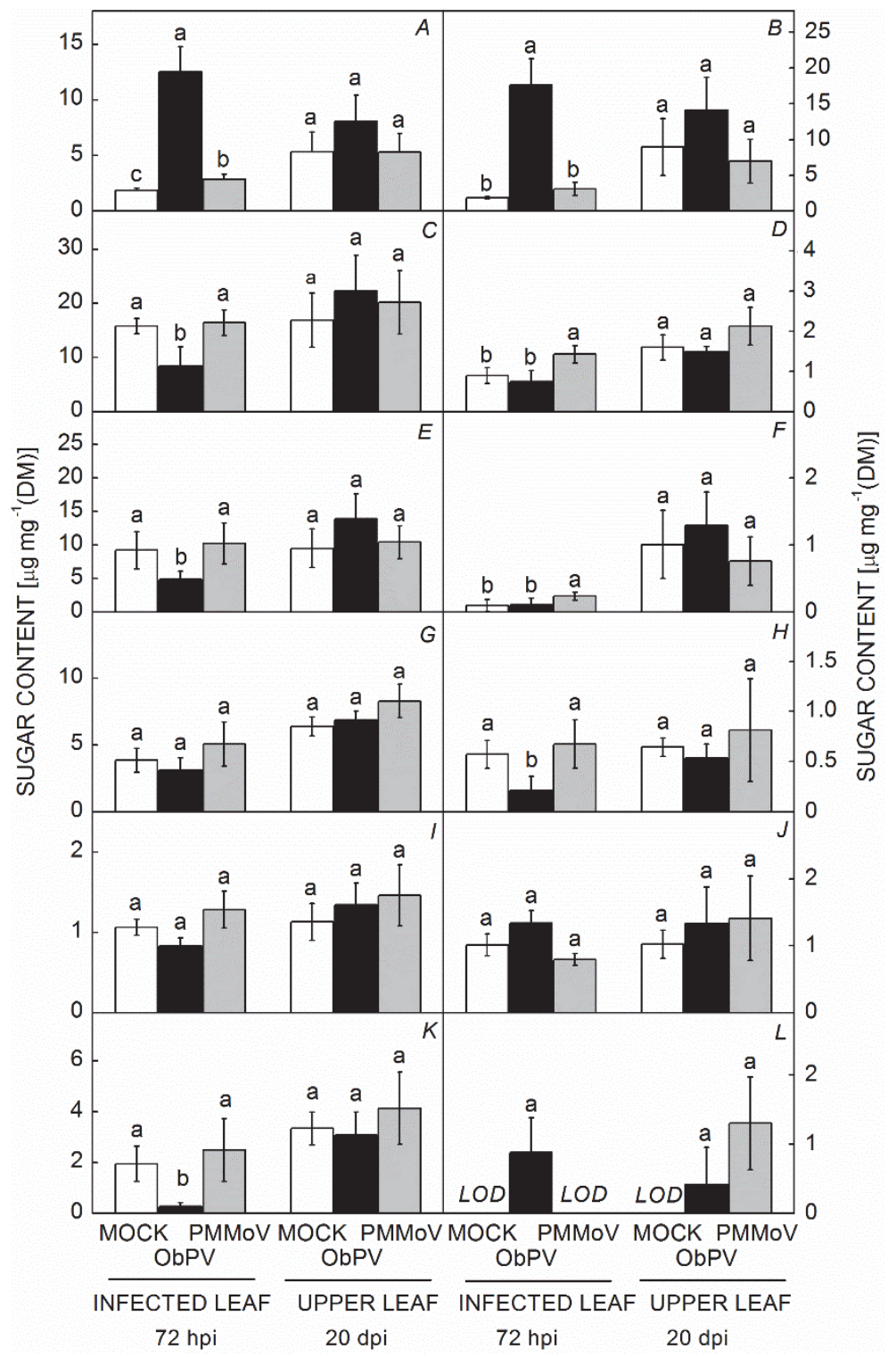

Fig. 5. Effects of ObPV and PMMoV inoculations on the content of soluble sugars and sugar alcohols in the inoculated, lower leaves as well as in the systemic, uninfected upper leaves of pepper plants. $(A)-$ glucose; $(B)$ - fructose; $(C)$ - sucrose; $(D)$ - maltose; $(E)$ - trehalose; $(F)$ - raffinose; $(G)$ - 1-kestose; $(H)$ - nystose; $(I)$ - inositol; $(J)$ - glycerol; $(K)$ maltoheptose; $(L)$ - glucose-6-phosphate. Local and systemic effects of virus inoculations were investigated 72 hours after inoculation (hpi) and 20 days after inoculation (dpi), respectively. Values marked with the same letter are not significantly different according to Duncan's test $(P \leq 0.05)$. LOD too low to detect. 


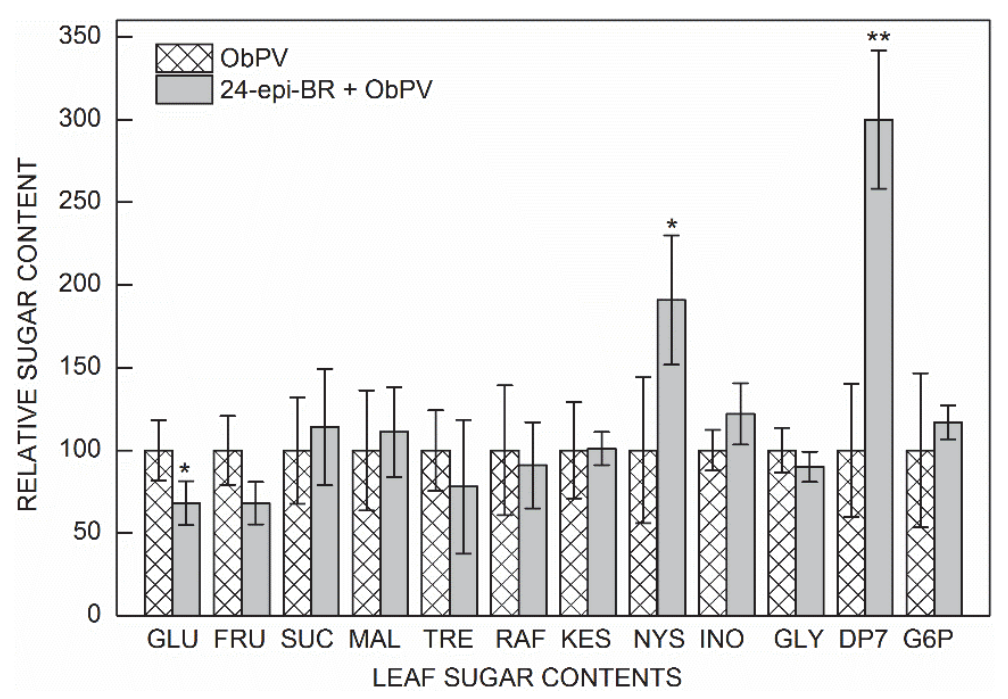

Fig. 6. Effect of 24-epibrassinolide (24-epi-BR) on the contents of sugars and sugar alcohols in ObPVinoculated pepper leaves 72 hours after inoculation (hpi). Values of ObPV-infected plants are taken as $100 \%$. ${ }^{*}$ and $* *$ - significant differences between 24-epi-BR pretreated and nontreated ObPVinoculated leaves at $P=5 \%$ and $1 \%$, respectively (Student's test). GLU - glucose; FRU - fructose; SUC - sucrose; MAL - maltose; TRE - trehalose; RAF - raffinose; KES - 1-kestose; NYS - nystose; INO - inositol; GLY - glycerol; DP7 - maltoheptose; G6P - glucose-6-phosphate.
Change in Chl fluorescence signal is generally considered as a sensitive indicator of many types of stressors including pathogen infection (Skoczowski et al. 2011, Strasser et al. 2010, Goltsev et al. 2012). In this work, the JIP test model was applied in order to acquire information about changes in the light phase (PSII efficiency) in virusinfected plants. In our experiments, ObPV inoculation already at 48 hpi (just before the appearance of visible symptoms) significantly affected photosynthesis; decreased the amplitude of fluorescence emission, and modified the shape of the induction curves (data not shown). There was no increase in $F_{0}$ values, which would indicate a decrease of efficiency of energy transfer between pigment molecules in the antenna. Thus, ObPV did not disturb the energy flow within the antenna. Values of $F_{m}$ and $F_{v}$ were significantly lowered by ObPV inoculation. The change in $\mathrm{F}_{\mathrm{m}}$ indicates that not all electron acceptors in the PSII can be completely reduced. In turn, the lower values of $F_{v}$ indicate greater dissipation of energy as heat, which can be associated with damage to the thylakoid. Difficulties in the movement of electrons in ObPV-infected tissues were shown also by increased values of $\mathrm{M}_{\mathrm{o}} . \mathrm{M}_{\mathrm{o}}$ informs about the net rate of the RC closure, where trapping increases the number of closed $\mathrm{RC}$, while electron transport decreases it. Finally, a reduction in the normalized area $\left(\mathrm{S}_{\mathrm{m}}\right)$ and turnover number (N) in ObPV-inoculated leaves indicated a reduced pool of effectively functioning electron carriers in ETC. The increase in relative fluorescence $\mathrm{V}_{\mathrm{K}}$ in ObPV-infected lower leaves of pepper indicated a deceleration in the donation of electrons to $\mathrm{P}_{680}{ }^{+}$. Such disturbances occurred in ObPV-infected leaves and it may inform about the inhibition of $\mathrm{O}_{2}$-evolving complex. Furthermore, the values of $F_{v} / F_{o}$ informing about the effectiveness of water decomposition were also lowered in ObPV-inoculated plants. Negative disturbances in energy flow in PSII of ObPV-infected leaves can be concluded from all three parameters of quantum efficiencies which were significantly lower in comparison to MOCK-inoculated control.
Thus, all these data indicate that virus infection caused significant changes of photosynthesis (light phase) in a hypersensitive host before the appearance of visible symptoms. We found only slight changes of fluorescence parameters after PMMoV inoculation until $72 \mathrm{hpi}$, which is in accordance with the slower and weaker reaction of the susceptible host, and with our previous results, when we detected some significant changes only 96 hpi (Rys et al. 2014).

Recently, our knowledge significantly increased about BRs as stress hormones and about their regulatory roles during photosynthesis. As mentioned above, BRs increase the content of photosynthetic pigments and induce the activity of several enzymes involved in photosynthesis, sugar production and transport (Holá 2011).

In accordance with the stress-alleviating and photosynthesis-promoting effects of BRs, 24-epi-BR pretreatment significantly alleviated the negative consequences of ObPV inoculation on the PSII efficiency. Protective effect of 24-epi-BR on PSII in plants exposed to various stressors was previously noted in oilseed rape and barley (Janeczko et al. 2005, Janeczko et al. 2011). BRs, which are known to increase the activity of antioxidant enzymes in plants (Yuan et al. 2012), may help to neutralize ROS and to reduce the damage of PSII (Gururani et al. 2015). Furthermore, BRs (similary to sterols) may stabilize the structure of cell membranes (Grunwald 1974), and by this way decrease the damaging effect of stresses. Our earlier studies support this hypothesis, since BR treatment alleviated the electrolyte leakage (connected to membrane damage) in bacteriuminoculated leaves (Skoczowski et al. 2011). Furthermore, a differential scanning calorimetry analysis revealed that 24-epi-BR alters the thermodynamic parameters of photosynthetic membranes (Dobrikova et al. 2014). In addition, transmission electron microscopic studies proved that thylakoids and chloroplasts maintained their typical shapes in salt-stressed cucumber plants due to the application of 24-epi-BR (Yuan et al. 2012). Finally, it is 
noticeable that BR-deficient barley mutants produce less fructooligosaccharide (nystose) than that of wild type plants (Janeczko et al. 2016). Nystose possesses membrane-stabilizing properties (Livingston et al. 2009), and in our experiment, the exogenous supplementation of 24-epi-BR led to a 2-fold increase of nystose content in ObPV-inoculated leaves, which may contribute to the protection of thylakoid membranes.

In order to obtain further information about the functioning of the photosynthetic apparatus, we carried out leaf reflectance investigations in virus-inoculated leaves. ObPV inoculation resulted in a substantial drop in the PRI index in infected pepper leaves (Fig. 3), that indicated low light-use efficiency and suggested changes in the functioning of the xanthophyll cycle. The PRI value is known to be influenced by the deepoxidation stage of the xanthophyll cycle, by the presence of zeaxanthin and by the radiation-use efficiency (Filella et al. 1996). The xanthophyll cycle is located in thylakoid membranes and it is one of the mechanisms that protects PS against photoinhibition (Demming-Adams and Adams 1996). This is in agreement with our results obtained using fluorescence analysis, where lower values of $F_{v}$ indicated a greater dissipation of energy as heat in ObPV-inoculated leaves in comparison to MOCK-inoculated control ones (Table 1). Decreasing SIPI values in both ObPV- and PMMoV-inoculated pepper leaves after $48 \mathrm{hpi}$ indicated a stress-induced disorder in the Car:Chl ratio. Increases in concentrations of Car relative to $\mathrm{Chl}$ are often observed when plants are subjected to stress (Peñuelas et al. 1994). In our previous work, decreased Chl $a$ and increased Car contents were observed in the ObPV-inoculated pepper leaves (Rys et al. 2014). ObPV inoculation led to loss of turgidity in infected leaves (Rys et al. 2014), which was confirmed by the decreasing WBI values observed in the present study (Fig. 3). Even slight loss of water content in leaf decreased the efficiency of photosynthesis. As a consequence of water loss due to ObPV inoculation, decreased $g_{\mathrm{s}}$ and low $E$ were also observed (Fig. 4). The very low $P_{\mathrm{N}}$ during ObPV infection was accompanied by increased $C_{\mathrm{i}}$ (Fig. 4), which may indicate that $P_{\mathrm{N}}$ was diminished not only by stomatal limitation, but also by biochemical factors. According to our previous work, ObPV inoculation led to elevated heat emission due to the enhanced metabolism of pepper leaves, mainly respiration (Rys et al. 2014). In accordance, the respiration rate of Cucumber mosaic virus-inoculated melon leaves was almost twice of that of control plants, resulting in a massive reduction of $P_{\mathrm{N}}$ values that were close to zero (Shalitin and Wolf 2000). However, the gross photosynthetic rate that indicates the level of carboxylation was similar in CMV-inoculated and control leaves.

It is known that infections by viruses can elicit changes of sugar concentrations in plants (Shalitin and Wolf 2000, Herbers et al. 2000). The sugar analysis of virus-infected pepper leaves revealed that the contents of two monosaccharides, glucose and fructose, massively increased, while that of sucrose significantly decreased in ObPVinoculated pepper leaves $72 \mathrm{hpi}$ (Fig. 5). These data suggest that invertase enzyme(s) were activated by ObPV inoculation. Invertase isoenzymes are also known to participate in signalling processes in infected plants (Herbers and Sonnewald 1998, Tauzin and Giardina 2014). Altered carbohydrate metabolism and partitioning was observed also in transgenic tobacco plants overexpressing the movement protein coding gene of Tobacco mosaic virus. Contents of sucrose, glucose, fructose, and starch were elevated in source leaves of transgenic tobacco plants (Lucas et al. 1993). The accumulated monosaccharides may be necessary as a source of carbon and energy for defense responses. In addition, genetic analyses have revealed extensive interactions between sugar and plant hormone signalling (Rolland et al. 2006). All these results suggest that sugars act as signals to activate or intensify immune reactions (Moghaddam and Van den Ende 2012). Our experiments also showed a massive accumulation of glucose-6phosphate in ObPV- (72 hpi and 20 dpi) and PMMoV(20 dpi) inoculated pepper leaves, which suggests the activation of hexokinase(s) by these viruses. Hexokinases have been identified as core components in plant sugar sensing and signalling (Rolland et al. 2006).

In addition, the present study revealed the presence of two fructooligosaccharides, 1-kestose and nystose, in pepper leaves. Fructans are water soluble polymers of fructose that possess antistress properties and serve as osmoprotectants (Hendry 1993, Livingston and Henson 1998). They stabilize cell membranes by inserting polysaccharides into the lipid-head regions of membranes (Livingston et al. 2009). ObPV inoculation significantly decreased the amount of nystose in the infected leaves. This phenomenon may be a consequence of the diminished sucrose content in ObPV-inoculated leaves (Müller et al. 2000). Due to protective properties of fructans, their decreasing cellular content may compromise the antistress mechanisms of plants. Interestingly, a pretreatment with 24-epi-BR prior to ObPV inoculation markedly elevated the foliar nystose content but did not influence significantly the kestose concentration. It is noteworthy that BR-deficient barley mutants produced less nystose than that of wild type plants (Janeczko et al. 2016).

ObPV inoculations markedly decreased the foliar contents of maltoheptose (a maltooligosaccharide containing seven units of glucose) and trehalose in the infected leaves (Fig. 5). Large accumulation of maltooligosaccharides occurs during starch degradation (Critchley et al. 2001), which may suggest that starch decomposition was hindered in ObPV-infected leaves. Interestingly, pretreatment with 24-epi-BR prior to ObPV inoculation counteracted the decrease of maltoheptose in the infected leaves (Fig. 6). The high accumulation of glucose and glucose-6-phosphate in ObPV-inoculated leaves suggests disturbances in trehalose biosynthesis. Trehalose can act as a ROS scavenger and it can stabilize cell membranes, 
therefore its lower cellular concentration may deteriorate plant stress resistance (Elbein et al. 2003).

It is noteworthy that in our studies PMMoV inoculation markedly induced the foliar contents of glucose, maltose, and raffinose in the infected leaves $72 \mathrm{hpi}$. According to Kaplan and Guy (2004), temperature stress led to maltose accumulation in chloroplast stroma. Maltose acted as osmoprotectant and had positive effects on the functionality of proteins, membranes, and membrane-associated processes. Raffinose has ROS scavenging capacity that may help to counteract oxidative stress of various origins (Elsayed et al. 2014).

Sugar and sugar alcohol concentrations in the upper, noninfected systemic leaves were not influenced $20 \mathrm{dpi}$ neither by ObPV nor by PMMoV inoculation except glucose-6-phosphate, which is noticeable since we could not detect this compound 72 hpi in PMMoV-infected leaves (Fig. 5). Probably systemic spreading of the virus into the upper leaves induced the synthesis of glucose-6phosphate.
In conclusion, pepper leaves showed markedly different physiological reactions in the incompatible, hypersensitive pepper-ObPV interaction as compared to the compatible, systemic pepper - PMMoV interaction. ObPV inoculation decreased the photosynthetic efficiency already $48 \mathrm{hpi}$, before the appearance of visible symptoms, and diminished the stomatal conductance, the transpiration rate, and the sucrose concentration. On the other hand, in accordance with the very low rate of net photosynthesis and decreased sucrose content, ObPV increased the intracellular $\mathrm{CO}_{2}$ concentration, as well as the glucose and fructose contents. The compatible PMMoV infection exerted much weaker or even insignificant effects on the photosynthetic parameters, spectral reflectance and gas exchange than that of ObPV. In PMMoV-inoculated pepper leaves, mainly the contents of "protective" sugars, such as raffinose and maltose, increased. It is noteworthy that pretreatment with the plant hormone 24-epi-BR prior to ObPV inoculation significantly alleviated the damage by the virus, mainly by the improvement of PSII efficiency.

Open Access This article is distributed under the terms of the Creative Commons Attribution License which permits any use, distribution, and reproduction in any medium, provided the original author(s) and the source are credited.

\section{References}

Almási A., Harsányi A., Gáborjányi R.: Photosynthetic alterations of virus infected plants. - Acta Phytopathol. Hun. 36: 1529, 2001.

Balachandran S., Osmond C.B., Makino A.: Effects of two strains of tobacco mosaic virus on photosynthetic characteristics and nitrogen partitioning in leaves of Nicotiana tabacum $c v$ Xanthi during photoacclimation under two nitrogen nutrition regimes. - Plant Physiol. 104: 1043-1050, 1994.

Baron M., Rahoutei J., Lazaro J. et al.: Photosystem II response to biotic and abiotic stress. - In: Mathis P. (ed.): Photosynthesis from Light to Biosphere. Pp. 897-901. Kluwer Academic Publishers, The Hague 1995.

Critchley J.H., Zeeman S.C., Takaha T. et al.: A critical role for disproportionating enzyme in starch breakdown is revealed by a knock-out mutation in Arabidopsis. - Plant J. 26: 89-100, 2001.

Dobrikova A.G., Vladkova R.S., Rashkov G.D. et al.: Effects of exogenous 24-epibrassinolide on the photosynthetic membranes under non-stress conditions. - Plant Physiol. Bioch. 80: 75-82, 2014.

Demmig-Adams B., Adams W.W. III: The role of xanthophyll cycle carotenoids in the protection of photosynthesis. - Trends Plant Sci. 1: 21-26, 1996

Elbein A.D., Pan Y.T., Pastuszak I. et al.: New insights on trehalose: a multifunctional molecule. - Glycobiology 13: 17R27R, 2003.

ElSayed A.I., Rafudeen M.S., Golldack D.: Physiological aspects of raffinose family oligosaccharides in plants: protection against abiotic stress. - Plant Biol. 16: 1-8, 2014.

Filella I., Amaro T., Araus J.L. et al.: Relationship between photosynthetic radiation-use efficiency of barley canopies and the photochemical reflectance index (PRI). - Physiol. Plantarum 96: 211-216, 1996.

Funayama S., Sonoike K., Terashima I.: Photosynthetic properties of leaves of Eupatorium makinoi infected by a geminivirus. - Photosynth. Res. 52: 253-261, 1997.

Gamon J.A., Peñuelas J., Field C.B.: A narrow-waveband spectral index that tracks diurnal changes in photosynthetic efficiency. - Remote Sens. Environ. 41: 35-44, 1992.

Gamon J.A., Serrano L., Surfus J.: The photochemical reflectance index: an optical indicator of photosynthetic radiation-use efficiency across species, functional types and nutrient levels. - Oecologia 112: 492-501, 1997.

Goltsev V., Zaharieva I., Chernev P. et al.: Drought-induced modifications of photosynthetic electron transport in intact leaves: analysis and use of neural networks as a tool for a rapid non-invasive estimation. - Biochim. Biophys. Acta 1817: 1490-1498, 2012.

Gonçalves M. C., Vega J., Oliveira J.G. et al.: Sugarcane yellow leaf virus infection leads to alterations in photosynthetic efficiency and carbohydrate accumulation in sugarcane leaves. - Fitopatol. Bras. 30: 10-16, 2005.

Gómez-Ariza J., Campo S., Rufat M. et al.: Sucrose-mediated priming of plant defence responses and broad-spectrum disease resistance by overexpression of the maize pathogenesis-related PRms protein in rice plants. - Mol. Plant Microbe In. 20: 832842, 2007.

Grunwald C.: Sterol molecular modifications influencing membrane permeability. - Plant Physiol. 54: 624-628, 1974.

Guo Y.P., Zhou H.F., Zeng G.H. et al.: [Effects of high temperature stress on photosynthetic rate and photosystem II activity in citrus.] - Chin. J. Appl. Ecol. 14: 867-870, 2003. [In Chinese]

Gururani M. A., Venkatesh J., Tran L.S.P.: Regulation of photosynthesis during abiotic stress-induced photoinhibition. Mol. Plant 8: 1304-1320, 2015.

Hendry G.A.F. Evolutionary origins and natural functions of fructans - a climatological, biogeographic and mechanistic appraisal. - New Phytol. 123: 3-14, 1993. 
Herbers K., Meuwly P., Métraux J.P.: Salicylic acid-independent induction of pathogenesis-related protein transcripts by sugars is dependent on leaf developmental stage. - FEBS Lett. 397: 239-244, 1996.

Herbers K., Sonnewald U.: Altered gene expression brought about by inter- and intracellularly formed hexoses and its possible implications for plant-pathogen interactions. - J. Plant Res. 111: 323-328, 1998.

Herbers K., Takahata Y., Melzer M. et al:: Regulation of carbohydrate partitioning during the interaction of potato virus $Y$ with tobacco. - Mol. Plant Pathol. 1: 51-59, 2000.

Holá D.: Brassinosteroids and photosynthesis. - In: Hayat S., Ahmad A. (ed.): Brassinosteroids: a Class of Plant Hormone. Pp. 143-192. Springer, Dordrecht, Heidelberg, New York 2011.

Janeczko A., Kościelniak J., Pilipowicz M. et al.: Protection of winter rape photosystem II by 24-epibrassinolide under cadmium stress. - Photosynthetica 43: 293-298, 2005.

Janeczko A., Biesaga-Kościelniak J., Oklešt'ková J. et al.: Role of 24-epibrassinolide in wheat production: physiological effects and uptake. - J. Agron. Crop Sci. 196: 311-321, 2010.

Janeczko A., Oklešt'ková J., Pociecha E. et al.: Physiological effects and transport of 24-epibrassinolide in heat-stressed barley. - Acta Physiol. Plant. 33: 1249-1259, 2011.

Janeczko A., Gruszka D., Pociecha E. et al.: Physiological and biochemical characterisation of watered and drought-stressed barley mutants in the $H v D W A R F$ gene encoding C6-oxidase involved in brassinosteroid biosynthesis. - Plant Physiol. Bioch. 99: 126-141, 2016.

Juhász C., Tóbiás I., Ádám A.L. et al.: Pepper 9- and 13lipoxygenase genes are differentially activated by two tobamoviruses and by hormone treatments. - Physiol. Mol. Plant P. 92: 59-69, 2015.

Kaplan F., Guy C.L.: Beta-Amylase induction and the protective role of maltose during temperature shock. - Plant Physiol. 135: 1674-1684, 2004

Knipling E.B.: Physical and physiological basis for the reflectance of visible and near-infrared radiation from vegetation. - Remote Sens. Environ. 1: 155-159, 1970.

Koch K.E.: Carbohydrate-modulated gene expression in plants. - Annu. Rev. Plant Phys. 47: 509-540, 1996.

Krezhova D., Dikova B., Maneva S.: Ground based hyperspectral remote sensing for disease detection of tobacco plants. - Bulg. J. Agric. Sci. 20: 1142-1150, 2014.

Livingston D.P. III, Henson C.A.: Apoplastic sugars, fructans, fructan exohydrolase, and invertase in winter oat: responses to second-phase cold hardening. - Plant Physiol. 116: 403-408, 1998.

Livingston D.P. III, Hincha D.K., Heyer A.G.: Fructan and its relationship to abiotic stress tolerance in plants. - Cell. Mol. Life Sci. 66: 2007-2023, 2009.

Lucas W.J., Olesinski A., Hull R.J. et al.: Influence of the tobacco mosaic virus $30-\mathrm{kDa}$ movement protein on carbon metabolism and photosynthate partitioning in transgenic tobacco plants. - Planta 190: 88-96, 1993.

Moghaddam B.M.R, van den Ende W.: Sugars and plant innate immunity. - J. Exp. Bot. 63: 3989-3998, 2012.

Müller J., Aeschbacher R.A., Sprenger N. et al.: Disaccharidemediated regulation of sucrose: fructan-6-fructosyltransferase, a key enzyme of fructan synthesis in barley leaves. - Plant Physiol. 123: 265-274, 2000.

Papageorgiou G.C., Tsimilli-Michael M., Stamatakis K.: The fast and slow kinetics of chlorophyll $a$ fluorescence induction in plants, algae and cyanobacteria: a viewpoint. - Photosynth.
Res. 94: 275-290, 2007.

Peñuelas J., Filella I., Biel C. et al.: The reflectance at the 950$970 \mathrm{~nm}$ region as an indicator of plant water status. - Int. J. Remote Sens. 14: 1887-1905, 1993.

Peñuelas J., Gamon J.A., Fredeen A.L. et al.: Reflectance indices associated with physiological changes in nitrogen-and waterlimited sunflower leaves. - Remote Sens. Environ. 48: 135146, 1994.

Peñuelas J., Filella I., Baret F.: Semi-empirical indices to assess carotenoids/chlorophyll $a$ ratio from leaf spectra reflectance. Photosynthetica 31: 221-230, 1995a.

Peñuelas J., Filella I., Gamon J.A.: Assessment of photosynthetic radiation-use efficiency with spectral reflectance. - New Physiol. 131: 291-296, 1995b.

Peñuelas J., Isla R., Filella I. et al.: Visible and near-infrared reflectance assessment of salinity effects on barley. - Crop Sci. 37: 198-202, 1997.

Pérez-Bueno M.L., Rahoutei J., Sajnani C. et al.: Proteomic analysis of the oxygen-evolving complex of photosystem II under biotec stress: Studies on Nicotiana benthamiana infected with tobamoviruses. - Proteomics 4: 418-425, 2004.

Rahoutei J., Barón M., García-Luque I. et al.: Effect of tobamovirus infection on thermoluminescence characteristics of chloroplasts from infected plants. - Z. Naturforsch. C 54: 634-639, 1999.

Rahoutei J., García-Luque I., Barón M.: Inhibition of photosynthesis by viral infection: effect on PSII structure and function. - Physiol. Plantarum 110: 286-292, 2000.

Roitsch T., Balibrea M.E., Hofmann M. et al.: Extracellular invertase: key metabolic enzyme and PR protein. - J. Exp. Bot 54: 513-524, 2003.

Rolland F., Baena-Gonzalez E., Sheen J.: Sugar sensing and signaling in plants: conserved and novel mechanisms. - Annu. Rev. Plant Biol. 57: 675-709, 2006.

Rys M., Juhász C., Surowka E. et al.: Comparison of a compatible and an incompatible pepper-tobamovirus interaction by biochemical and non-invasive techniques: chlorophyll $a$ fluorescence, isothermal calorimetry and FT-Raman spectroscopy. - Plant Physiol. Bioch. 83: 267-278, 2014.

Ryšlavá H., Müller K., Semorádová S. et al.: Photosynthesis and activity of phosphoenolpyruvate carboxylase in Nicotiana tabacum L. leaves infected by Potato virus A and Potato virus Y. - Photosynthetica 41: 357-363, 2003.

Shalitin D., Wolf S.: Cucumber mosaic virus infection affects sugar transport in melon plants. - Plant Physiol. 123: 597-604, 2000

Skoczowski A., Janeczko A., Gullner G. et al.: Response of brassinosteroid-treated oilseed rape cotyledons to infection with the wild type and HR-mutant of Pseudomonas syringae or with P. fluorescens. - J. Therm. Anal. Calorim. 104: 131-139, 2011.

Smeekens S., Ma J., Hanson J. et al.: Sugar signals and molecular networks controlling plant growth. - Curr. Opin. Plant Biol. 13: 274-279, 2010.

Solovchenko A.: Quantification of screening pigments and their efficiency in situ. - In: Solovchenko A. (ed.): Photoprotection in Plants. Pp. 119-141. Springer Series in Biophysics 14, Springer-Verlag, Berlin, Heidelberg 2010.

Strasser R.J., Srivastava A., Tsimilli-Michael M.: The fluorescence transient as a tool to characterize and screen photosynthetics samples. - In: Yunus M., Pathre U., Mohanty P. (ed.): Probing Photosynthesis: Mechanism, Regulation and Adaptation. Pp. 445-483. Taylor and Francis, London 2000.

Strasser R.J., Tsimilli-Michael M., Qiang S. et al.: Simultaneous 
in vivo recording of prompt and delayed fluorescence and 820$\mathrm{nm}$ reflection changes during drying and after rehydration of the resurrection plant Haberlea rhodopensis. - Biochim. Biophys. Acta 1797: 1313-1326, 2010.

Tauzin A.S., Giardina T.: Sucrose and invertases, a part of the plant defense response to the biotic stresses. - Front. Plant Sci. 5: 293, 2014.

Tóbiás I., Fraser R.S.S., Gerwitz A.: The gene-for-gene relationship between Capsicum annum L. and tobacco mosaic virus: effects on virus multiplication, ethylene synthesis and accumulation of pathogenesis-related proteins. - Physiol. Mol. Plant Pathol. 35: 271-286, 1989.

Velasco L., Janssen D., Ruiz-Garcia L. et al.: The complete nucleotide sequence and development of a diferential detection assay for a pepper mild mottle virus (PMMoV) isolate that overcomes $L^{3}$ resistance in pepper. - J. Virol. Methods 106: 135-140, 2002

Wang Z-Y.: Brassinosteroids modulate plant immunity at multiple levels. - P. Natl. Acad. Sci. USA 109: 7-8, 2012.

Yuan L., Shu S., Sun J. et al.: Effects of 24-epibrassinolide on the photosynthetic characteristics, antioxidant system, and chloroplast ultrastructure in Cucumis sativus L. under $\mathrm{Ca}\left(\mathrm{NO}_{3}\right)_{2}$ stress. - Photosynth. Res. 112: 205-214, 2012.

Yusuf M., Fariduddin Q., Ahmad I. et al.: Brassinosteroidmediated evaluation of antioxidant system and nitrogen metabolism in two contrasting cultivars of Vigna radiata under different levels of nickel. - Physiol. Mol. Biol. Plants 20: 449460, 2014. 